\title{
THE GENERALIZED KINETIC EQUATION FOR SYMMETRIC PARTICLE SYSTEMS
}

\author{
HALYNA M. HUBAL
}

\begin{abstract}
The generalized kinetic equation is obtained for symmetric system of many particles interacting via a pair potential. A representation of a solution of the Cauchy problem for the BBGKY hierarchy is used in the form of an expansion over particle groups whose evolution is governed by the cumulants (semi-invariants).
\end{abstract}

\section{Introduction}

The evolution of states of many-particle systems is described by the BBGKY hierarchy of equations [1], [3]. A solution of the Cauchy problem for the BBGKY hierarchy of equations can be represented in the form of the iteration or the functional series, or the non-equilibrium cluster expansion: [3], [6], [7]. In this article we use a representation of a solution in the form of an expansion over particle groups whose evolution is governed by the cumulants (semiinvariants) of the evolution operator of the corresponding particle group [5]. Such a representation of solution enables us to describe the cluster nature of the evolution of infinite particle systems with different symmetry properties in detail.

In certain situations states of many-particle systems can be described in terms of the one-particle distribution function that satisfies some closed evolution equation, which we call the kinetic equation.

In this article, we derive the generalized kinetic equation in explicit form from the BBGKY hierarchy of equations. For a mathematical formulation of the problem we consider the Cauchy problem for the BBGKY hierarchy of equations with initial data which are products of one-particle distribution functions. Such an assumption for the initial data is natural for the kinetic description of a gas, since its states, in this case, are described only by the oneparticle distribution function. Under this assumption we prove that the Cauchy problem for the BBGKY hierarchy of equations in the space of sequences of summable functions is equivalent to the corresponding initial value problem for the generalized kinetic equation.

Received 28 September 2010. 
Note that generalized kinetic equations were obtained for a discrete velocity symmetric system of hard spheres [2] and for a symmetric system of particles [4] by using a solution of the Cauchy problem for the BBGKY hierarchy in another representation. For a symmetric system of particles the kinetic equation has been obtained in non-explicit form: see [3].

In Sections 2-4, after having given the formulation of the problem we present the main technical result (Theorem 4.1) which is necessary to derive, in Section 5, the generalized kinetic equation (Theorem 5.1). In Section 6 we formulate the existence theorem (Theorem 6.1) for the derived kinetic equation. In Section 7 we formulate conclusion.

\section{The BBGKY hierarchy of equations}

Let us consider a symmetric system of finitely many particles of mass $m=1$ interacting via a pair potential $\Phi$. We assume that the interaction potential $\Phi$ satisfies conditions guaranteeing the existence an uniqueness of solutions, global in time, for the initial value problem for the Hamilton equations of a system of an arbitrary finite number of particles. For example, $\Phi$ is a twice continuously differentiable function with a compact support.

Each $i$-th particle is characterized by the phase-coordinates $\left(q_{i}, p_{i}\right) \equiv x_{i} \in$ $\mathrm{R}^{v} \times \mathrm{R}^{v}, v \geq 1$.

Let $L^{1}\left(\mathrm{R}^{\nu s} \times \mathrm{R}^{\nu s}\right)$ be the linear space of summable functions $f_{s}\left(x_{1}, \ldots, x_{s}\right)$ defined on the phase space $\mathrm{R}^{v s} \times \mathrm{R}^{v s}$ and invariant under permutations of the arguments $\left(x_{1}, \ldots, x_{s}\right)$ with the norm

$$
\left\|f_{s}\right\|=\int_{\mathrm{R}^{v s} \times \mathrm{R}^{v s}} d x_{1} \ldots d x_{s}\left|f_{s}\left(x_{1}, \ldots, x_{s}\right)\right| .
$$

We define the space $L_{0}^{1}\left(\mathrm{R}^{v s} \times \mathrm{R}^{v s}\right)$ as consisting of those functions $f_{s} \in$ $L^{1}\left(\mathrm{R}^{v s} \times \mathrm{R}^{v s}\right)$ which have compact support, and which are continuously differentiable with respect to the variables $\left(x_{1}, \ldots, x_{s}\right)$. This space is dense in $L^{1}\left(\mathrm{R}^{\nu s} \times \mathrm{R}^{\nu s}\right)$. Let $\alpha>1$ be an integer. By

$$
L_{\alpha}^{1}:=\bigoplus_{s=0}^{\infty} \alpha^{s} L^{1}\left(\mathrm{R}^{v s} \times \mathrm{R}^{v s}\right)
$$

we denote the Banach space of infinite sequences $f=\left\{f_{s}\left(x_{1}, \ldots, x_{s}\right)\right\}_{s \geq 0}$ with the property that $\|f\|:=\sum_{s=0}^{\infty} \alpha^{s}\left\|f_{s}\right\|<\infty$.

The state of such a system is determined by a solution of the Cauchy problem 
for the BBGKY hierarchy of equations:

$$
\begin{aligned}
\frac{\partial}{\partial t} F_{s}\left(t, x_{1}, \ldots, x_{s}\right)= & \left\{H_{s}\left(x_{1}, \ldots, x_{s}\right), F_{s}\left(t, x_{1}, \ldots, x_{s}\right)\right\} \\
& +\frac{1}{v} \int_{\mathrm{R}^{v} \times \mathrm{R}^{v}} d x_{s+1}\left\{\sum_{i=1}^{s} \Phi\left(q_{i}-q_{s+1}\right), F_{s+1}\left(t, x_{1}, \ldots, x_{s+1}\right)\right\}
\end{aligned}
$$

with initial data possessing the factorization property (the chaos property):

$$
\begin{aligned}
\left.F_{1}\left(t, x_{1}\right)\right|_{t=0} & =F_{1}\left(0, x_{1}\right), \\
\left.F_{s}\left(t, x_{1}, \ldots, x_{s}\right)\right|_{t=0} & =\prod_{i=1}^{s} F_{1}\left(0, x_{i}\right), \quad s \geq 2,
\end{aligned}
$$

where $H_{s}\left(x_{1}, \ldots, x_{s}\right)$ is the Hamilton function, $\frac{1}{v}$ is the density, $\{\cdot, \cdot\}$ is the Poisson bracket [3], $F_{1}\left(0, x_{i}\right) \in L_{0}^{1}\left(\mathrm{R}^{\nu} \times \mathrm{R}^{\nu}\right)$.

\section{A global solution of the Cauchy problem for the BBGKY hierarchy}

A solution of the Cauchy problem for the BBGKY hierarchy of equations is represented as the expansion over particle groups whose evolution is governed by the cumulants [5]

$$
F_{|Y|}(t, Y)=\sum_{n=0}^{\infty} \frac{1}{n !} \int_{\mathrm{R}^{v n} \times \mathrm{R}^{\mathrm{v} n}} d(X \backslash Y) \mathfrak{A}_{\left|X_{Y}\right|}\left(t, X_{Y}\right) F_{|X|}(0, X),
$$

where

$$
\begin{gathered}
Y=\left(x_{1}, \ldots, x_{s}\right), \quad X=\left(x_{1}, \ldots, x_{s}, x_{s+1}, \ldots, x_{s+n}\right), \\
X_{Y}=\left(Y, x_{s+1}, \ldots, x_{s+n}\right), \quad d(X \backslash Y)=d x_{s+1} \ldots d x_{s+n}, \quad d x_{j}=d q_{j} d p_{j}, \\
\mathfrak{A}_{\left|X_{Y}\right|}\left(t, X_{Y}\right)=\sum_{P: X_{Y}=\cup_{i} X_{i}}(-1)^{|P|-1}(|P|-1) ! \prod_{X_{i} \subset P} S_{\left|X_{i}\right|}\left(-t, X_{i}\right), \quad|X \backslash Y| \geq 0 .
\end{gathered}
$$

Here $\sum_{P: X_{Y}=\cup_{i} X_{i}}$ is the sum over all possible partitions of the set $X_{Y}$ into $|P|$ nonempty pairwise disjoint subsets $X_{i} \subset X_{Y}$ and the set $Y$ lies in one of the subsets $X_{i}$. The symbol $S_{s}(-t)$ is the evolution operator:

$$
\begin{aligned}
& \left(S_{s}(-t) f_{s}\right)\left(x_{1}, \ldots, x_{s}\right) \\
& \quad=f_{s}\left(X_{1}\left(-t, x_{1}, \ldots, x_{s}\right), \ldots, X_{s}\left(-t, x_{1}, \ldots, x_{s}\right)\right), \quad s \geq 1,
\end{aligned}
$$

where $X_{i}(t)=X_{i}\left(t, x_{1}, \ldots, x_{s}\right), i=1, \ldots, s$ is a solution of the initial value problem for the Hamilton equations of $s$-particle system with initial data $X_{i}\left(0, x_{1}, \ldots, x_{s}\right)=x_{i}, i=1, \ldots, s[3]$. 
Taking into account (3) for the Cauchy problem (1), (2) in the space $L_{\alpha}^{1}$ the following theorem is true.

THEOREM 3.1. If $F_{1}(0) \in L_{0}^{1}\left(\mathrm{R}^{\nu} \times \mathrm{R}^{\nu}\right) \subset L^{1}\left(\mathrm{R}^{\nu} \times \mathrm{R}^{\nu}\right)$ then there exists a unique strong, global in time, solution $F(t)=\left\{F_{s}(t, Y)\right\}_{s=|Y| \geq 0}$, where $F_{s}(t) \in L^{1}\left(\mathrm{R}^{v s} \times \mathrm{R}^{v s}\right), s \geq 1$, of the Cauchy problem (1), (2), which is given as the expansion over particle groups whose evolution is governed by the cumulants

$$
\begin{aligned}
& \text { (4) } F_{S}(t, Y)=S_{s}(-t, Y) \prod_{i=1}^{s} F_{1}\left(0, x_{i}\right) \\
& +\sum_{n=1}^{\infty} \frac{1}{v^{n}} \frac{1}{n !} \int_{\mathrm{R}^{v n} \times \mathrm{R}^{v n}} d(X \backslash Y) \mathfrak{A}_{\left|X_{Y}\right|}\left(t, X_{Y}\right) \prod_{i=1}^{n+s} F_{1}\left(0, x_{i}\right), \quad s \geq 1 .
\end{aligned}
$$

The proof of this statement is straightforward. Each term of the series in (4) is a well-defined function, since the integrand is defined almost everywhere outside of the set $U_{s+n}^{0}$ of zero Lebesgue measure [3]. The functional series (4) converges in the norm of the space $L^{1}\left(\mathrm{R}^{\nu s} \times \mathrm{R}^{\nu s}\right)$ for arbitrary $t \in \mathrm{R}^{1}$. The following estimate holds

$$
\left\|F_{s}(t)\right\| \leq\left\|F_{1}(0)\right\|^{s} e^{\frac{1}{v}\left\|F_{1}(0)\right\|} \frac{1}{1-\frac{\left\|F_{1}(0)\right\|}{v}}, \quad s \geq 1 .
$$

Indeed, denote by $k$ the number of subsets $X_{i}$ in partition $P$ and taking into account that $\left\|S_{\left|X_{i}\right|}(-t)\right\|=1$ in the space of summable functions we obtain

$$
\begin{aligned}
\left\|F_{S}(t)\right\| \leq & \sum_{n=0}^{\infty} \frac{1}{v^{n}} \frac{1}{n !} \| \int_{\mathrm{R}^{v n} \times \mathrm{R}^{v n}} d(X \backslash Y) \sum_{k=1}^{n+1} \sum_{P:|P|=k}(-1)^{k-1} \\
& \times(k-1) ! \prod_{X_{i} \subset P} S_{\left|X_{i}\right|}\left(-t, X_{i}\right) \prod_{i=1}^{n+s} F_{1}\left(0, x_{i}\right) \| \\
\leq & \sum_{n=0}^{\infty} \frac{1}{v^{n}} \frac{1}{n !} \int_{\mathrm{R}^{v(n+s)} \times \mathrm{R}^{v(n+s)}} d X \sum_{k=1}^{n+1} C_{n}^{k-1}(k-1) !\left|\prod_{i=1}^{n+s} F_{1}\left(0, x_{i}\right)\right| \\
= & \sum_{n=0}^{\infty} \frac{1}{v^{n}} \frac{1}{n !} \sum_{k=1}^{n+1} C_{n}^{k-1}(k-1) !\left\|F_{1}(0)\right\|^{n+s} \\
= & \sum_{n=0}^{\infty} \frac{1}{v^{n}} \sum_{j=0}^{n} \frac{1}{(n-j) !}\left\|F_{1}(0)\right\|^{n+s}
\end{aligned}
$$




$$
\begin{aligned}
& =\sum_{n=0}^{\infty} \frac{1}{v^{n}} \sum_{j=0}^{n} \frac{1}{j !}\left\|F_{1}(0)\right\|^{n+s}=\sum_{j=0}^{\infty} \frac{1}{j !} \sum_{n=j}^{\infty} \frac{1}{v^{n}}\left\|F_{1}(0)\right\|^{n+s} \\
& =\left\|F_{1}(0)\right\|^{s} \sum_{j=0}^{\infty} \frac{1}{j !}\left(\frac{\left\|F_{1}(0)\right\|}{v}\right)^{j} \sum_{n=j}^{\infty}\left(\frac{\left\|F_{1}(0)\right\|}{v}\right)^{n-j} \\
& =\left\|F_{1}(0)\right\|^{s} e^{\frac{\left\|F_{1}(0)\right\|}{v}} \frac{1}{1-\frac{\left\|F_{1}(0)\right\|}{v}} .
\end{aligned}
$$

The symbol $C_{n}^{k-1}$ stands for a binomial coefficient.

\section{On a solution of the nonlinear equation for the one-particle distribution function}

If the initial data (2) are assigned in terms of the initial one-particle distribution function $F_{1}(0)$ then the problem (1), (2) is not a "completely well-defined" in the sense, that the initial data $F_{s}(0)=\prod_{i=1}^{s} F_{1}\left(0, x_{i}\right), s \geq 2$, are not independent for every unknown function in (1). Thus, let us re-formulate the problem (1), (2) as a new Cauchy problem for the independent unknown function, i.e. $F_{1}(t)$, together with an infinite sequence of functionals, $F_{s}\left(t \mid F_{1}(t)\right)=$ $F_{s}\left(t, Y \mid F_{1}(t)\right), s=|Y| \geq 2$ (see Eq. (24) and text below).

Let us consider relation (4) for $s=1$ as a closed equation for the function $F_{1}(0)$ in the space $L^{1}\left(\mathrm{R}^{v} \times \mathrm{R}^{\nu}\right)$

$$
F_{1}(0)=A F_{1}(0)
$$

where

$$
\begin{aligned}
& \left(A F_{1}(0)\right)\left(x_{1}\right)=S_{1}\left(t, x_{1}\right) F_{1}\left(t, x_{1}\right) \\
& -\sum_{n=1}^{\infty} \frac{1}{v^{n}} \frac{1}{n !} \int_{\mathrm{R}^{v n} \times \mathrm{R}^{v n}} d\left(X \backslash\left\{x_{1}\right\}\right) S_{1}\left(t, x_{1}\right) \mathfrak{A}_{\mid X_{\left\{x_{1}\right\}}(}\left(t, X_{\left\{x_{1}\right\}}\right) \prod_{i=1}^{n+1} F_{1}\left(0, x_{i}\right),
\end{aligned}
$$

$d\left(X \backslash\left\{x_{1}\right\}\right)=d x_{2} \ldots d x_{n+1}$.

Denote $S_{1}\left(t, x_{1}\right) F_{1}\left(t, x_{1}\right) \equiv F_{1}^{0}$ and let $\left\|F_{1}\left(t, x_{1}\right)\right\| \leq r<+\infty$. Then $F_{1}^{0} \in L^{1}\left(\mathrm{R}^{v} \times \mathrm{R}^{v}\right),\left\|F_{1}^{0}\right\|=\left\|S_{1}\left(t, x_{1}\right) F_{1}\left(t, x_{1}\right)\right\| \leq r<+\infty$. In the space $L_{0}^{1}\left(\mathrm{R}^{v} \times \mathrm{R}^{v}\right) \subset L^{1}\left(\mathrm{R}^{v} \times \mathrm{R}^{v}\right)$ we consider a ball

$$
\mathrm{S}\left(F_{1}^{0}, R\right) \equiv\left\{F_{1}(0) \in L_{0}^{1}\left(\mathrm{R}^{\nu} \times \mathrm{R}^{\nu}\right):\left\|F_{1}(0)-F_{1}^{0}\right\| \leq R\right\} .
$$

The following theorem holds. 
THEOREM 4.1. Let $v, R$, and $r$ be given strictly positive numbers. Let $x$ and $z$ be real solutions of the equations

$$
\frac{e^{x}}{1-x}=\frac{2 R+r}{R+r}, \quad \text { and } \quad e^{z} \cdot \frac{1+z-z^{2}}{(1-z)^{2}}=2
$$

respectively. Suppose that

$$
\frac{1}{v}<\frac{1}{R+r} \min \{x, z\}
$$

Put $\hat{\mathfrak{A}}_{\left|X_{i}\right|}\left(t, X_{i}\right)=\mathfrak{A}_{\left|X_{i}\right|}\left(t, X_{i}\right) \prod_{x_{j} \in X_{i}} S_{1}\left(t, x_{j}\right)$. Then there exist a unique solution of equation (6) in the domain $\mathrm{S}\left(F_{1}^{0}, R\right) \subset L^{1}\left(\mathrm{R}^{v} \times \mathrm{R}^{v}\right)$ given by the formula

$$
\begin{aligned}
& F_{1}\left(0, x_{1}\right)=S_{1}\left(t, x_{1}\right) F_{1}\left(t, x_{1}\right) \\
& +\sum_{n=1}^{\infty} \frac{1}{v^{n}} \int_{\mathrm{R}^{v n} \times \mathrm{R}^{v n}} d\left(X \backslash\left\{x_{1}\right\}\right) \tilde{\mathfrak{A}}^{(n)}(t) \prod_{i=1}^{n+1} F_{1}\left(t, x_{i}\right),
\end{aligned}
$$

where

$$
\begin{aligned}
\tilde{\mathfrak{U}}^{(1)}(t)= & -S_{1}\left(t, x_{1}\right) \hat{\mathfrak{U}}_{2}\left(t, x_{1}, x_{2}\right), \\
\tilde{\mathfrak{U}}^{(2)}(t)= & S_{1}\left(t, x_{1}\right) \hat{\mathfrak{A}}_{2}\left(t, x_{1}, x_{2}\right)\left(\hat{\mathfrak{U}}_{2}\left(t, x_{1}, x_{3}\right)+\hat{\mathfrak{A}}_{2}\left(t, x_{2}, x_{3}\right)\right) \\
& \quad-\frac{1}{2 !} S_{1}\left(t, x_{1}\right) \hat{\mathfrak{U}}_{3}\left(t, x_{1}, x_{2}, x_{3}\right),
\end{aligned}
$$

and so on.

Proof. Let us establish that the operator $A$ defined in (7) maps the ball $\mathrm{S}\left(F_{1}^{0}, R\right)$ into itself and is a contraction operator. Since

$$
\left\|F_{1}(0)\right\| \leq\left\|F_{1}(0)-F_{1}^{0}\right\|+\left\|F_{1}^{0}\right\| \leq R+r,
$$

then by definition (7) and the condition $\left\|S_{\left|X_{i}\right|}\left(t, X_{i}\right)\right\|=1$ in the space of summable functions we have

$$
\begin{aligned}
& \left\|A F_{1}(0)-F_{1}^{0}\right\| \\
& \quad=\left\|\sum_{n=1}^{\infty} \frac{1}{v^{n}} \frac{1}{n !} \int_{\mathrm{R}^{v n} \times \mathrm{R}^{v n}} d\left(X \backslash\left\{x_{1}\right\}\right) S_{1}\left(t, x_{1}\right) \mathfrak{A}_{\left|X_{\left\{x_{1} \mid\right.}\right|}\left(t, X_{\left\{x_{1}\right\}}\right) \prod_{i=1}^{n+1} F_{1}\left(0, x_{i}\right)\right\| \\
& \quad \leq \sum_{n=1}^{\infty} \frac{1}{v^{n}} \frac{1}{n !} \int_{\mathrm{R}^{v(n+1)} \times \mathrm{R}^{v(n+1)}} d X \sum_{k=1}^{n+1} C_{n}^{k-1}(k-1) !\left|\prod_{i=1}^{n+1} F_{1}\left(0, x_{i}\right)\right|
\end{aligned}
$$




$$
\begin{aligned}
& =\sum_{n=1}^{\infty} \frac{1}{v^{n}} \frac{1}{n !} \sum_{k=1}^{n+1} C_{n}^{k-1}(k-1) !\left\|F_{1}(0)\right\|^{n+1} \\
& =\left\|F_{1}(0)\right\|\left(\sum_{n=0}^{\infty} \frac{1}{v^{n}} \sum_{j=0}^{n} \frac{1}{j !}\left\|F_{1}(0)\right\|^{n}-1\right) \\
& =\left\|F_{1}(0)\right\|\left(\sum_{j=0}^{\infty} \frac{1}{j !} \sum_{n=j}^{\infty}\left(\frac{\left\|F_{1}(0)\right\|}{v}\right)^{n}-1\right) \\
& =\left\|F_{1}(0)\right\|\left(\sum_{j=0}^{\infty} \frac{1}{j !}\left(\frac{\left\|F_{1}(0)\right\|}{v}\right)^{j} \sum_{n=j}^{\infty}\left(\frac{\left\|F_{1}(0)\right\|}{v}\right)^{n-j}-1\right) \\
& =\left\|F_{1}(0)\right\|\left(e^{\frac{\left\|F_{1}(0)\right\|}{v}} \frac{1}{1-\frac{\left\|F_{1}(0)\right\|}{v}}-1\right) \leq(R+r)\left(\frac{1}{1-\frac{R+r}{v}} e^{\frac{R+r}{v}}-1\right) .
\end{aligned}
$$

Thus, the operator $A$ maps the ball $\mathrm{S}\left(F_{1}^{0}, R\right)$ into itself if

$$
(R+r)\left(\frac{1}{1-\frac{R+r}{v}} e^{\frac{R+r}{v}}-1\right) \leq R .
$$

Let us find a simple condition on the density $\frac{1}{v}$ in order that (9) holds. Observe that (9) is equivalent to

$$
\frac{1}{1-\frac{R+r}{v}} e^{\frac{R+r}{v}} \leq \frac{2 R+r}{R+r}
$$

and let $x \in \mathrm{R}$ be such that

$$
\frac{e^{x}}{1-x}=\frac{2 R+r}{R+r}
$$

Then for $v$ such that $\frac{R+r}{v} \leq x$ the inequality in (9) follows. Here we use the fact that the function $y \mapsto \frac{e^{y}}{1-y}$ is increasing for $y<1$.

Thus, the condition (9) gives the condition on the density

$$
\frac{1}{v} \leq \frac{1}{R+r} x
$$

where $x$ is a solution of the equation (10). 
Let us find a condition under which the operator $A$ is a contraction on $\mathrm{S}\left(F_{1}^{0}, R\right)$. For arbitrary elements $Y_{1}, Y_{2} \in \mathrm{S}\left(F_{1}^{0}, R\right)$ we have

(11) $\left\|A Y_{1}-A Y_{2}\right\| \leq \sum_{n=1}^{\infty} \frac{1}{v^{n}} \frac{1}{n !} \sum_{k=1}^{n+1} C_{n}^{k-1}(k-1)$ !

$$
\times \int_{\mathrm{R}^{v(n+1)} \times \mathrm{R}^{v(n+1)}} d X\left|\prod_{j=1}^{n+1} Y_{1}\left(0, x_{j}\right)-\prod_{j=1}^{n+1} Y_{2}\left(0, x_{j}\right)\right| .
$$

Let us formulate a version of Duhamel's formula as the following lemma.

\section{Lemma 4.2. The equality}

$$
\begin{aligned}
& \prod_{j=1}^{n+1} Y_{1}\left(0, x_{j}\right)-\prod_{j=1}^{n+1} Y_{2}\left(0, x_{j}\right) \\
& \quad=\sum_{i=1}^{n+1} \prod_{j=1}^{i-1} Y_{1}\left(0, x_{j}\right)\left(Y_{1}\left(0, x_{i}\right)-Y_{2}\left(0, x_{i}\right)\right) \prod_{j=i+1}^{n+1} Y_{2}\left(0, x_{j}\right), \quad n \in \mathrm{N}
\end{aligned}
$$

is true.

Proof. Let us use induction on the number of particles. It is evident that for $n=1$ the equality (12) is true. Assume that the equality (12) is true for $n=m$. Let us prove the equality (12) for $n=m+1$, using the assumption:

$$
\begin{aligned}
\prod_{j=1}^{m+2} Y_{1}\left(0, x_{j}\right)-\prod_{j=1}^{m+2} Y_{2}\left(0, x_{j}\right) & \\
= & \prod_{j=1}^{m+1} Y_{1}\left(0, x_{j}\right)\left(Y_{1}\left(0, x_{m+2}\right)-Y_{2}\left(0, x_{m+2}\right)\right) \\
& \quad+\left(\prod_{j=1}^{m+1} Y_{1}\left(0, x_{j}\right)-\prod_{j=1}^{m+1} Y_{2}\left(0, x_{j}\right)\right) Y_{2}\left(0, x_{m+2}\right) \\
= & \sum_{i=1}^{m+2} \prod_{j=1}^{i-1} Y_{1}\left(0, x_{j}\right)\left(Y_{1}\left(0, x_{i}\right)-Y_{2}\left(0, x_{i}\right)\right) \prod_{j=i+1}^{m+2} Y_{2}\left(0, x_{j}\right) .
\end{aligned}
$$

Thus, the equality (12) is true for $n \in \mathrm{N}$. 
Using Lemma 4.2 the expression (11) takes the form

$$
\begin{aligned}
\| A Y_{1}- & A Y_{2} \| \\
\leq & \sum_{n=1}^{\infty} \frac{1}{v^{n}} \frac{1}{n !} \sum_{k=1}^{n+1} C_{n}^{k-1}(k-1) ! \\
& \quad \times\left\|\sum_{i=1}^{n+1} \prod_{j=1}^{i-1} Y_{1}\left(0, x_{j}\right)\left(Y_{1}\left(0, x_{i}\right)-Y_{2}\left(0, x_{i}\right)\right) \prod_{j=i+1}^{n+1} Y_{2}\left(0, x_{j}\right)\right\| \\
\leq & \sum_{n=1}^{\infty} \frac{1}{v^{n}} \frac{1}{n !} \sum_{k=1}^{n+1} C_{n}^{k-1}(k-1) ! \sum_{i=1}^{n+1}(R+r)^{i-1}\left\|Y_{1}-Y_{2}\right\|(R+r)^{n+1-i} \\
= & \sum_{n=1}^{\infty} \frac{1}{v^{n}} \frac{1}{n !} \sum_{k=1}^{n+1} C_{n}^{k-1}(k-1) !(n+1)(R+r)^{n}\left\|Y_{1}-Y_{2}\right\| \\
= & \sum_{n=1}^{\infty}(n+1)\left(\frac{R+r}{v}\right)^{n} \sum_{j=0}^{n} \frac{1}{j !}\left\|Y_{1}-Y_{2}\right\| .
\end{aligned}
$$

A condition which makes the operator $A$ a strict contraction is of the form

$$
\sum_{n=0}^{\infty}(n+1)\left(\frac{R+r}{v}\right)^{n} \sum_{j=0}^{n} \frac{1}{j !}<2 .
$$

We want to rewrite the condition on the density $\frac{1}{v}$ in inequality (13). Therefore we notice that, for $|w|<1$,

$$
\begin{aligned}
\sum_{n=0}^{\infty}( & +1) w^{n} \sum_{j=0}^{n} \frac{1}{j !} \\
= & \sum_{j=0}^{\infty} \sum_{n=j}^{\infty} \frac{(n+1) w^{n}}{j !}=\sum_{j=0}^{\infty} \frac{1}{j !} \frac{d}{d w}\left(\sum_{n=j}^{\infty} w^{n+1}\right) \\
& =\frac{d}{d w}\left(\sum_{j=0}^{\infty} \frac{1}{j !} \frac{w^{j+1}}{1-w}\right)=\frac{d}{d w}\left(e^{w} \frac{w}{1-w}\right)=e^{w} \cdot \frac{1+w-w^{2}}{(1-w)^{2}} .
\end{aligned}
$$

Choose $0<z<1$ in such a way that

$$
e^{z} \cdot \frac{1+z-z^{2}}{(1-z)^{2}}=2
$$

and put $w=\frac{R+r}{v}$. If $0<w<z$, then the inequality (13) is satisfied and so $A$ is a strict contraction for such a density $\frac{1}{v}$. 
Thus, the condition (13) gives the following condition on the density

$$
\frac{1}{v}<\frac{1}{R+r} z
$$

where $z$ is a solution of the equation (14).

Thus, under condition

$$
\frac{1}{v}<\frac{1}{R+r} \min \{x, z\}
$$

where $x$ is a solution of the equation (10), $z$ is a solution of the equation (14), there exists a unique solution of equation (6) in the domain $\mathrm{S}\left(F_{1}^{0}, R\right) \subset$ $L^{1}\left(\mathrm{R}^{v} \times \mathrm{R}^{v}\right)$. This solution can be determined as the limit of successive approximations $F_{1}^{(n)}(0)=A F_{1}^{(n-1)}(0)$, where $F_{1}^{(0)}(0)=F_{1}^{0} \equiv S_{1}(t) F_{1}(t)$. The limit $\lim _{n \rightarrow \infty} F_{1}^{(n)}(0)=F_{1}(0)$ has the form (8).

According to (7) the first approximation of the solution, which describes the interaction between particles is expressed by the following formula

$$
\begin{aligned}
& F_{1}^{(1)}(0)=A F_{1}^{0} \\
& =F_{1}^{0}-\frac{1}{v} \int_{\mathrm{R}^{v} \times \mathrm{R}^{v}} d x_{2} S_{1}\left(t, x_{1}\right) \mathfrak{A}_{2}\left(t, x_{1}, x_{2}\right) \prod_{i=1}^{2} S_{1}\left(t, x_{i}\right) F_{1}\left(t, x_{i}\right) \\
& -\frac{1}{v^{2}} \frac{1}{2 !} \int_{\mathrm{R}^{2 v} \times \mathrm{R}^{2 v}} d x_{2} d x_{3} S_{1}\left(t, x_{1}\right) \mathfrak{A}_{3}\left(t, x_{1}, x_{2}, x_{3}\right) \prod_{i=1}^{3} S_{1}\left(t, x_{i}\right) F_{1}\left(t, x_{i}\right)-\cdots
\end{aligned}
$$

Analogously the second approximation of the solution, which describes the interaction between particles, is expressed by the following formula

$$
\begin{aligned}
F_{1}^{(2)}(0)= & A F_{1}^{(1)}(0) \\
= & F_{1}^{0}-\frac{1}{v} \int_{\mathrm{R}^{v} \times \mathrm{R}^{v}} d x_{2} S_{1}\left(t, x_{1}\right) \mathfrak{A}_{2}\left(t, x_{1}, x_{2}\right) \prod_{i=1}^{2} S_{1}\left(t, x_{i}\right) F_{1}\left(t, x_{i}\right) \\
& +\frac{1}{v^{2}} \int_{\mathrm{R}^{2 v} \times \mathrm{R}^{2 v}} d x_{2} d x_{3}\left(S_{1}\left(t, x_{1}\right) \mathfrak{A}_{2}\left(t, x_{1}, x_{2}\right) S_{1}\left(t, x_{1}\right)\right. \\
& \times \mathfrak{A}_{2}\left(t, x_{1}, x_{3}\right) \prod_{i=1}^{3} S_{1}\left(t, x_{i}\right) F_{1}\left(t, x_{i}\right) \\
& +S_{1}\left(t, x_{1}\right) \mathfrak{A}_{2}\left(t, x_{1}, x_{2}\right) S_{1}\left(t, x_{2}\right) \mathfrak{A}_{2}\left(t, x_{2}, x_{3}\right) \prod_{i=1}^{3} S_{1}\left(t, x_{i}\right) F_{1}\left(t, x_{i}\right) \\
& \left.-\frac{1}{2 !} S_{1}\left(t, x_{1}\right) \mathfrak{U}_{3}\left(t, x_{1}, x_{2}, x_{3}\right) \prod_{i=1}^{3} S_{1}\left(t, x_{i}\right) F_{1}\left(t, x_{i}\right)\right)+\cdots
\end{aligned}
$$


In a similar manner we construct the $n$-th approximation.

Denote

$$
\begin{aligned}
& \tilde{\mathfrak{U}}^{(1)}(t)=-S_{1}\left(t, x_{1}\right) \mathfrak{U}_{2}\left(t, x_{1}, x_{2}\right) \prod_{i=1}^{2} S_{1}\left(t, x_{i}\right)=-S_{1}\left(t, x_{1}\right) \hat{\mathfrak{A}}_{2}\left(t, x_{1}, x_{2}\right), \\
& \tilde{\mathfrak{U}}^{(2)}(t)=S_{1}\left(t, x_{1}\right) \mathfrak{A}_{2}\left(t, x_{1}, x_{2}\right) \\
& \times\left(S_{1}\left(t, x_{1}\right) \mathfrak{A}_{2}\left(t, x_{1}, x_{3}\right) \prod_{i=1}^{3} S_{1}\left(t, x_{i}\right)\right. \\
& \left.+S_{1}\left(t, x_{2}\right) \mathfrak{U}_{2}\left(t, x_{2}, x_{3}\right) \prod_{i=1}^{3} S_{1}\left(t, x_{i}\right)\right) \\
& -\frac{1}{2 !} S_{1}\left(t, x_{1}\right) \mathfrak{P}_{3}\left(t, x_{1}, x_{2}, x_{3}\right) \prod_{i=1}^{3} S_{1}\left(t, x_{i}\right) \\
& =S_{1}\left(t, x_{1}\right) \hat{\mathfrak{P}}_{2}\left(t, x_{1}, x_{2}\right)\left(\hat{\mathfrak{V}}_{2}\left(t, x_{1}, x_{3}\right)+\hat{\mathfrak{V}}_{2}\left(t, x_{2}, x_{3}\right)\right) \\
& -\frac{1}{2 !} S_{1}\left(t, x_{1}\right) \hat{\mathfrak{U}}_{3}\left(t, x_{1}, x_{2}, x_{3}\right) \text {, }
\end{aligned}
$$

and so on, where $\hat{\mathfrak{A}}_{\left|X_{i}\right|}\left(t, X_{i}\right)=\mathfrak{A}_{\left|X_{i}\right|}\left(t, X_{i}\right) \prod_{x_{j} \in X_{i}} S_{1}\left(t, x_{j}\right)$. Then we obtain

$$
\begin{aligned}
F_{1}\left(0, x_{1}\right)= & S_{1}\left(t, x_{1}\right) F_{1}\left(t, x_{1}\right)+\frac{1}{v} \int_{\mathrm{R}^{v} \times \mathrm{R}^{v}} d x_{2} \tilde{\mathfrak{Y}}^{(1)}(t) \prod_{i=1}^{2} F_{1}\left(t, x_{i}\right) \\
& \quad+\frac{1}{v^{2}} \int_{\mathrm{R}^{2 v} \times \mathrm{R}^{2 v}} d x_{2} d x_{3} \tilde{\mathfrak{U}}^{(2)}(t) \prod_{i=1}^{3} F_{1}\left(t, x_{i}\right)+\cdots \\
= & S_{1}\left(t, x_{1}\right) F_{1}\left(t, x_{1}\right) \\
& +\sum_{n=1}^{\infty} \frac{1}{v^{n}} \int_{\mathrm{R}^{v n} \times \mathrm{R}^{v n}} d\left(X \backslash\left\{x_{1}\right\}\right) \tilde{\mathfrak{U}}^{(n)}(t) \prod_{i=1}^{n+1} F_{1}\left(t, x_{i}\right) .
\end{aligned}
$$

\section{The generalized kinetic equation}

The one-particle distribution function which is a solution of the initial value problem (1), (2) can be represented as the following expansion (Theorem 3.1):

$$
\begin{aligned}
F_{1}\left(t, x_{1}\right) & =S_{1}\left(-t, x_{1}\right) F_{1}\left(0, x_{1}\right) \\
+ & \sum_{n=1}^{\infty} \frac{1}{v^{n}} \frac{1}{n !} \int_{\mathrm{R}^{v n} \times \mathrm{R}^{v n}} d\left(X \backslash\left\{x_{1}\right\}\right) \mathfrak{A}_{\left|X_{\left\{x_{1}\right\}}\right|}\left(t, X_{\left\{x_{1}\right\}}\right) \prod_{i=1}^{n+1} F_{1}\left(0, x_{i}\right) .
\end{aligned}
$$


THEOREM 5.1. The strong derivative with respect to t of relation (15) has the form

$$
\text { (16) } \begin{aligned}
\frac{\partial}{\partial t} F_{1}\left(t, x_{1}\right)= & -p_{1} \frac{\partial}{\partial q_{1}} F_{1}\left(t, x_{1}\right) \\
& +\frac{1}{v} \int_{\mathrm{R}^{v} \times \mathrm{R}^{v}} d x_{2}\left\{\Phi\left(q_{1}-q_{2}\right), F_{2}\left(t, x_{1}, x_{2} \mid F_{1}(t)\right)\right\}+\cdots,
\end{aligned}
$$

where the functional $F_{2}\left(t, x_{1}, x_{2} \mid F_{1}(t)\right)$ is given by the following formula:

$$
\begin{aligned}
& \text { (17) } F_{2}\left(t, x_{1}, x_{2} \mid F_{1}(t)\right)=\hat{S}_{2}^{t}\left(x_{1}, x_{2}\right) \prod_{i=1}^{2} F_{1}\left(t, x_{i}\right) \\
& +\sum_{n=1}^{\infty} \frac{1}{v^{n}} \int_{\mathrm{R}^{v n} \times \mathrm{R}^{v n}} d\left(X \backslash\left\{x_{1}, x_{2}\right\}\right) \breve{\mathfrak{d}}^{(\mathrm{n})}\left(t, X_{\left\{x_{1}, x_{2}\right\}}\right) \prod_{i=1}^{n+2} F_{1}\left(t, x_{i}\right) .
\end{aligned}
$$

Here

$$
\begin{aligned}
\hat{S}_{2}^{t}\left(x_{1}, x_{2}\right)= & S_{2}\left(-t, x_{1}, x_{2}\right) \prod_{i=1}^{2} S_{1}\left(t, x_{i}\right), \\
\breve{\mathfrak{U}}^{(1)}(t)= & -\hat{S}_{2}^{t}\left(x_{1}, x_{2}\right)\left(\hat{\mathfrak{A}}_{2}\left(t, x_{1}, x_{3}\right)+\hat{\mathfrak{U}}_{2}\left(t, x_{2}, x_{3}\right)\right)+\hat{\mathfrak{U}}_{3}\left(t, x_{1}, x_{2}, x_{3}\right), \\
\breve{\mathfrak{U}}^{(2)}(t)= & \left(\hat{\mathfrak{U}}_{2}\left(t, x_{1}, x_{4}\right)+\hat{\mathfrak{U}}_{2}\left(t, x_{2}, x_{4}\right)+\hat{\mathfrak{U}}_{2}\left(t, x_{3}, x_{4}\right)\right) \\
& \times\left(\hat{S}_{2}^{t}\left(x_{1}, x_{2}\right)\left(\hat{\mathfrak{A}}_{2}\left(t, x_{1}, x_{3}\right)+\hat{\mathfrak{A}}_{2}\left(t, x_{2}, x_{3}\right)\right)-\hat{\mathfrak{U}}_{3}\left(t, x_{1}, x_{2}, x_{3}\right)\right) \\
& -\frac{1}{2 !} \hat{S}_{2}^{t}\left(x_{1}, x_{2}\right)\left(\hat{\mathfrak{U}}_{2}\left(t, x_{1}, x_{3}, x_{4}\right)+\hat{\mathfrak{U}}_{2}\left(t, x_{2}, x_{3}, x_{4}\right)\right) \\
& +\frac{1}{2 !} \hat{\mathfrak{U}}_{3}\left(t, x_{1}, x_{2}, x_{3}, x_{4}\right)
\end{aligned}
$$

and so on.

Proof. Let us consider relation (15) in the form

$$
F_{1}(t)=U(t) F_{1}(0),
$$

where

$$
\begin{aligned}
& \left(U(t) F_{1}(0)\right)\left(x_{1}\right)=S_{1}\left(-t, x_{1}\right) F_{1}\left(0, x_{1}\right) \\
& +\sum_{n=1}^{\infty} \frac{1}{v^{n}} \frac{1}{n !} \int_{\mathrm{R}^{v n} \times \mathrm{R}^{v n}} d\left(X \backslash\left\{x_{1}\right\}\right) \mathfrak{A}_{\mid X_{\left\{x_{1}\right\}}\left(t, X_{\left\{x_{1}\right\}}\right)} \prod_{i=1}^{n+1} F_{1}\left(0, x_{i}\right) .
\end{aligned}
$$


Thus, using the group property [5], [6] of the operator $U(t)$ and the expression (15) we obtain

$$
\begin{aligned}
\frac{\partial}{\partial t} & F_{1}\left(t, x_{1}\right) \\
= & \frac{\partial}{\partial t}\left(U(t) F_{1}(0)\right)\left(x_{1}\right) \\
= & \lim _{\Delta t \rightarrow 0} \frac{1}{\Delta t}\left((U(t+\Delta t)-U(t)) F_{1}(0)\right)\left(x_{1}\right) \\
= & \lim _{\Delta t \rightarrow 0} \frac{1}{\Delta t}\left(U(t)(U(\Delta t)-I) F_{1}(0)\right)\left(x_{1}\right) \\
= & \lim _{\Delta t \rightarrow 0} \frac{1}{\Delta t}\left((U(\Delta t)-I) U(t) F_{1}(0)\right)\left(x_{1}\right) \\
= & \lim _{\Delta t \rightarrow 0} \frac{1}{\Delta t}\left(S_{1}\left(-\Delta t, x_{1}\right)-I\right)\left(U(t) F_{1}(0)\right)\left(x_{1}\right) \\
& +\frac{1}{v} \lim _{\Delta t \rightarrow 0} \frac{1}{\Delta t} \int_{\mathbb{R}^{v} \times \mathrm{R}^{v}} d x_{2} \mathfrak{A}_{2}\left(\Delta t, x_{1}, x_{2}\right) U\left(t, x_{1}, x_{2}\right) \prod_{i=1}^{2} F_{1}\left(0, x_{i}\right)+\cdots
\end{aligned}
$$

Taking into account the equality [3]

$$
\lim _{\Delta t \rightarrow 0} \frac{1}{\Delta t}\left(S_{1}\left(-\Delta t, x_{1}\right)-I\right)=\left\{H_{1}, \cdot\right\},
$$

the expression [5]

$$
\mathfrak{H}_{2}\left(\Delta t, x_{1}, x_{2}\right)=S_{2}\left(-\Delta t, x_{1}, x_{2}\right)-S_{1}\left(-\Delta t, x_{1}\right) S_{1}\left(-\Delta t, x_{2}\right)
$$

and using the Liouville theorem, we obtain

$$
\begin{aligned}
\frac{\partial}{\partial t} F_{1}( & \left.t, x_{1}\right) \\
= & \left\{H_{1}, F_{1}\left(t, x_{1}\right)\right\} \\
& +\frac{1}{v} \lim _{\Delta t \rightarrow 0} \frac{1}{\Delta t}\left(\int_{\mathrm{R}^{v} \times \mathrm{R}^{v}} d x_{2}\left(S_{2}\left(-\Delta t, x_{1}, x_{2}\right)-I\right)\right. \\
& \left.-\left(S_{1}\left(-\Delta t, x_{1}\right)-I\right) \times \int_{\mathrm{R}^{v} \times \mathrm{R}^{v}} d x_{2}\right) F_{2}\left(t, x_{1}, x_{2} \mid F_{1}(t)\right)+\cdots \\
= & -p_{1} \frac{\partial}{\partial q_{1}} F_{1}\left(t, x_{1}\right) \\
& +\frac{1}{v}\left(\int_{\mathrm{R}^{v} \times \mathrm{R}^{v}} d x_{2}\left\{H_{2}, \cdot\right\}-\left\{H_{1}, \cdot\right\} \int_{\mathrm{R}^{v} \times \mathrm{R}^{v}} d x_{2}\right) F_{2}\left(t, x_{1}, x_{2} \mid F_{1}(t)\right)+\cdots
\end{aligned}
$$


Let us calculate the difference of the terms between the parentheses in the final of the equality in (18). Recall that the function $\Phi$ stands for the pair potential of our problem. A calculation shows:

$$
\begin{array}{rl}
\int_{\mathrm{R}^{v} \times \mathrm{R}^{v}} & d x_{2}\left\{H_{2}, F_{2}\left(t, x_{1}, x_{2} \mid F_{1}(t)\right)\right\} \\
= & \int_{\mathrm{R}^{v} \times \mathrm{R}^{v}} d x_{2}\left\{\sum_{i=1}^{2} \frac{p_{i}^{2}}{2}+\Phi\left(q_{1}-q_{2}\right), F_{2}\left(t, x_{1}, x_{2} \mid F_{1}(t)\right)\right\} \\
= & \int_{\mathrm{R}^{v} \times \mathrm{R}^{v}} d x_{2}\left\{\Phi\left(q_{1}-q_{2}\right), F_{2}\left(t, x_{1}, x_{2} \mid F_{1}(t)\right)\right\} \\
& -p_{1} \int_{\mathrm{R}^{v} \times \mathrm{R}^{v}} d x_{2} \frac{\partial}{\partial q_{1}} F_{2}\left(t, x_{1}, x_{2} \mid F_{1}(t)\right) \\
& \quad-\int_{\mathrm{R}^{v} \times \mathrm{R}^{v}} d x_{2} p_{2} \frac{\partial}{\partial q_{2}} F_{2}\left(t, x_{1}, x_{2} \mid F_{1}(t)\right) \\
= & \int_{\mathrm{R}^{v} \times \mathrm{R}^{v}} d x_{2}\left\{\Phi\left(q_{1}-q_{2}\right), F_{2}\left(t, x_{1}, x_{2} \mid F_{1}(t)\right)\right\} \\
& -p_{1} \frac{\partial}{\partial q_{1}} \int_{\mathrm{R}^{v} \times \mathrm{R}^{v}} d x_{2} F_{2}\left(t, x_{1}, x_{2} \mid F_{1}(t)\right) \\
& -\int_{-\infty}^{\infty} d p_{2} p_{2} \int_{-\infty}^{\infty} d q_{2} \frac{\partial}{\partial q_{2}} F_{2}\left(t, x_{1}, x_{2} \mid F_{1}(t)\right) \\
= & \int_{\mathrm{R}^{v} \times \mathrm{R}^{v}} d x_{2}\left\{\Phi\left(q_{1}-q_{2}\right), F_{2}\left(t, x_{1}, x_{2} \mid F_{1}(t)\right)\right\} \\
& \quad-p_{1} \frac{\partial}{\partial q_{1}} \int_{\mathrm{R}^{v} \times \mathrm{R}^{v}} d x_{2} F_{2}\left(t, x_{1}, x_{2} \mid F_{1}(t)\right) .
\end{array}
$$

In the ultimate equality of (19) we used the fact that the probability of state with infinite distance between particles is equal to zero:

$$
\int_{-\infty}^{\infty} d p_{2} p_{2} \int_{-\infty}^{\infty} d q_{2} \frac{\partial}{\partial q_{2}} F_{2}\left(t, x_{1}, x_{2} \mid F_{1}(t)\right)=0 .
$$

We also have

$$
\begin{aligned}
\left\{H_{1}, \int_{\mathrm{R}^{v} \times \mathrm{R}^{v}} d x_{2} F_{2}\left(t, x_{1}, x_{2} \mid F_{1}(t)\right)\right\} & \\
= & -p_{1} \frac{\partial}{\partial q_{1}} \int_{\mathrm{R}^{v} \times \mathrm{R}^{v}} d x_{2} F_{2}\left(t, x_{1}, x_{2} \mid F_{1}(t)\right) .
\end{aligned}
$$


By substituting (19) and (20) into (18) we obtain

$$
\begin{aligned}
\frac{\partial}{\partial t} F_{1}\left(t, x_{1}\right) & =-p_{1} \frac{\partial}{\partial q_{1}} F_{1}\left(t, x_{1}\right) \\
& +\frac{1}{v} \int_{\mathrm{R}^{v} \times \mathrm{R}^{v}} d x_{2}\left\{\Phi\left(q_{1}-q_{2}\right), F_{2}\left(t, x_{1}, x_{2} \mid F_{1}(t)\right)\right\}+\cdots
\end{aligned}
$$

The explicit construction of the functional $F_{2}\left(t, x_{1}, x_{2} \mid F_{1}(t)\right)$ goes as follows. The solution (4) for $Y=\left\{x_{1}, x_{2}\right\}$ has the form (22)

$$
\begin{aligned}
& F_{2}\left(t, x_{1}, x_{2}\right) \\
& =S_{2}\left(-t, x_{1}, x_{2}\right) \prod_{i=1}^{2} F_{1}\left(0, x_{i}\right) \\
& \quad+\frac{1}{v} \int_{\mathrm{R}^{v} \times \mathrm{R}^{v}} d x_{3} \mathfrak{A}_{3}\left(t, x_{1}, x_{2}, x_{3}\right) \prod_{i=1}^{3} F_{1}\left(0, x_{i}\right) \\
& \quad+\frac{1}{v^{2}} \frac{1}{2 !} \int_{\mathrm{R}^{2 v} \times \mathrm{R}^{2 v}} d x_{3} d x_{4} \mathfrak{A}_{4}\left(t, x_{1}, x_{2}, x_{3}, x_{4}\right) \prod_{i=1}^{4} F_{1}\left(0, x_{i}\right)+\cdots
\end{aligned}
$$

We rewrite the solution in (8) as follows:

(23)

$$
\begin{aligned}
& F_{1}\left(0, x_{1}\right) \\
& =S_{1}\left(t, x_{1}\right) F_{1}\left(t, x_{1}\right) \\
& \quad-\frac{1}{v} \int_{\mathrm{R}^{v} \times \mathrm{R}^{v}} d x_{2} S_{1}\left(t, x_{1}\right) \mathfrak{A}_{2}\left(t, x_{1}, x_{2}\right) \prod_{i=1}^{2} S_{1}\left(t, x_{i}\right) F_{1}\left(t, x_{i}\right) \\
& \quad+\frac{1}{v^{2}} \int_{\mathrm{R}^{2 v} \times \mathrm{R}^{2 v}} d x_{2} d x_{3}\left(S_{1}\left(t, x_{1}\right) \mathfrak{A}_{2}\left(t, x_{1}, x_{2}\right) S_{1}\left(t, x_{2}\right) \mathfrak{A}_{2}\left(t, x_{2}, x_{3}\right)\right. \\
& \quad+S_{1}\left(t, x_{1}\right) \mathfrak{A}_{2}\left(t, x_{1}, x_{2}\right) S_{1}\left(t, x_{1}\right) \mathfrak{H}_{2}\left(t, x_{1}, x_{3}\right) \\
& \left.\quad-\frac{1}{2 !} S_{1}\left(t, x_{1}\right) \mathfrak{A}_{3}\left(t, x_{1}, x_{2}, x_{3}\right)\right) \prod_{i=1}^{3} S_{1}\left(t, x_{i}\right) F_{1}\left(t, x_{i}\right)+\cdots
\end{aligned}
$$

By substituting the solution (23) into the solution (22), taking into account the 


\section{interaction between particles we obtain}

$$
\begin{aligned}
& F_{2}\left(t, x_{1}, x_{2} \mid F_{1}(t)\right) \\
& =S_{2}\left(-t, x_{1}, x_{2}\right)\left[\prod_{i=1}^{2} S_{1}\left(t, x_{i}\right) F_{1}\left(t, x_{i}\right)\right. \\
& -\frac{1}{v}\left(S_{1}\left(t, x_{2}\right) F_{1}\left(t, x_{2}\right) \int_{\mathrm{R}^{v} \times \mathrm{R}^{v}} d x_{3} S_{1}\left(t, x_{1}\right) \mathfrak{A}_{2}\left(t, x_{1}, x_{3}\right)\right. \\
& \times S_{1}\left(t, x_{1}\right) F_{1}\left(t, x_{1}\right) S_{1}\left(t, x_{3}\right) F_{1}\left(t, x_{3}\right) \\
& \left.+S_{1}\left(t, x_{1}\right) F_{1}\left(t, x_{1}\right) \int_{\mathrm{R}^{v} \times \mathrm{R}^{v}} d x_{3} S_{1}\left(t, x_{2}\right) \mathfrak{A}_{2}\left(t, x_{2}, x_{3}\right) \prod_{i=2}^{3} S_{1}\left(t, x_{i}\right) F_{1}\left(t, x_{i}\right)\right) \\
& +\frac{1}{v^{2}}\left(S _ { 1 } ( t , x _ { 1 } ) F _ { 1 } ( t , x _ { 1 } ) \int _ { \mathrm { R } ^ { 2 v } \times \mathrm { R } ^ { 2 v } } d x _ { 3 } d x _ { 4 } \left(S_{1}\left(t, x_{2}\right) \mathfrak{A}_{2}\left(t, x_{2}, x_{3}\right)\right.\right. \\
& \times S_{1}\left(t, x_{3}\right) \mathfrak{U}_{2}\left(t, x_{3}, x_{4}\right) \prod_{i=2}^{4} S_{1}\left(t, x_{i}\right) F_{1}\left(t, x_{i}\right) \\
& +S_{1}\left(t, x_{2}\right) \mathfrak{A}_{2}\left(t, x_{2}, x_{3}\right) S_{1}\left(t, x_{2}\right) \mathfrak{A}_{2}\left(t, x_{2}, x_{4}\right) \prod_{i=2}^{4} S_{1}\left(t, x_{i}\right) F_{1}\left(t, x_{i}\right) \\
& \left.-\frac{1}{2 !} S_{1}\left(t, x_{2}\right) \mathfrak{A}_{3}\left(t, x_{2}, x_{3}, x_{4}\right) \prod_{i=2}^{4} S_{1}\left(t, x_{i}\right) F_{1}\left(t, x_{i}\right)\right) \\
& +\int_{\mathrm{R}^{v} \times \mathrm{R}^{v}} d x_{3} S_{1}\left(t, x_{1}\right) \mathfrak{H}_{2}\left(t, x_{1}, x_{3}\right) S_{1}\left(t, x_{1}\right) F_{1}\left(t, x_{1}\right) S_{1}\left(t, x_{3}\right) F_{1}\left(t, x_{3}\right) \\
& \times \int_{\mathrm{R}^{v} \times \mathrm{R}^{v}} d x_{4} S_{1}\left(t, x_{2}\right) \mathfrak{U}_{2}\left(t, x_{2}, x_{4}\right) S_{1}\left(t, x_{2}\right) F_{1}\left(t, x_{2}\right) S_{1}\left(t, x_{4}\right) F_{1}\left(t, x_{4}\right) \\
& +\int_{\mathrm{R}^{v} \times \mathrm{R}^{v}} d x_{3} S_{1}\left(t, x_{2}\right) \mathfrak{A}_{2}\left(t, x_{2}, x_{3}\right) \prod_{i=2}^{3} S_{1}\left(t, x_{i}\right) F_{1}\left(t, x_{i}\right) \\
& \times \int_{\mathrm{R}^{v} \times \mathrm{R}^{v}} d x_{4} S_{1}\left(t, x_{1}\right) \mathfrak{U}_{2}\left(t, x_{1}, x_{4}\right) S_{1}\left(t, x_{1}\right) F_{1}\left(t, x_{1}\right) S_{1}\left(t, x_{4}\right) F_{1}\left(t, x_{4}\right) \\
& +S_{1}\left(t, x_{2}\right) F_{1}\left(t, x_{2}\right) \int_{\mathrm{R}^{2 v} \times \mathrm{R}^{2 v}} d x_{3} d x_{4}\left(S_{1}\left(t, x_{1}\right) \mathfrak{H}_{2}\left(t, x_{1}, x_{3}\right)\right. \\
& \times S_{1}\left(t, x_{3}\right) \mathfrak{A}_{2}\left(t, x_{3}, x_{4}\right) S_{1}\left(t, x_{1}\right) F_{1}\left(t, x_{1}\right) \prod_{i=3}^{4} S_{1}\left(t, x_{i}\right) F_{1}\left(t, x_{i}\right) \\
& +S_{1}\left(t, x_{1}\right) \mathfrak{H}_{2}\left(t, x_{1}, x_{3}\right) S_{1}\left(t, x_{1}\right) \mathfrak{A}_{2}\left(t, x_{1}, x_{4}\right) \\
& \times S_{1}\left(t, x_{1}\right) F_{1}\left(t, x_{1}\right) \prod_{i=3}^{4} S_{1}\left(t, x_{i}\right) F_{1}\left(t, x_{i}\right)
\end{aligned}
$$




$$
\begin{aligned}
& \left.\left.\left.-\frac{1}{2 !} S_{1}\left(t, x_{1}\right) \mathfrak{A}_{3}\left(t, x_{1}, x_{3}, x_{4}\right) S_{1}\left(t, x_{1}\right) F_{1}\left(t, x_{1}\right) \prod_{i=3}^{4} S_{1}\left(t, x_{i}\right) F_{1}\left(t, x_{i}\right)\right)\right)\right] \\
& +\frac{1}{v} \int_{\mathrm{R}^{v} \times \mathrm{R}^{v}} d x_{3} \mathfrak{U}_{3}\left(t, x_{1}, x_{2}, x_{3}\right)\left[\prod_{i=1}^{3} S_{1}\left(t, x_{i}\right) F_{1}\left(t, x_{i}\right)\right. \\
& -\frac{1}{v} \prod_{i=1}^{2} S_{1}\left(t, x_{i}\right) F_{1}\left(t, x_{i}\right) \int_{\mathrm{R}^{v} \times \mathrm{R}^{v}} d x_{4} S_{1}\left(t, x_{3}\right) \mathfrak{A}_{2}\left(t, x_{3}, x_{4}\right) \\
& \times \prod_{i=3}^{4} S_{1}\left(t, x_{i}\right) F_{1}\left(t, x_{i}\right) \\
& -\frac{1}{v} \prod_{i=2}^{3} S_{1}\left(t, x_{i}\right) F_{1}\left(t, x_{i}\right) \int_{\mathrm{R}^{v} \times \mathrm{R}^{v}} d x_{4} S_{1}\left(t, x_{1}\right) \mathfrak{A}_{2}\left(t, x_{1}, x_{4}\right) \\
& \times S_{1}\left(t, x_{1}\right) F_{1}\left(t, x_{1}\right) S_{1}\left(t, x_{4}\right) F_{1}\left(t, x_{4}\right) \\
& -\frac{1}{v} S_{1}\left(t, x_{1}\right) F_{1}\left(t, x_{1}\right) S_{1}\left(t, x_{3}\right) F_{1}\left(t, x_{3}\right) \\
& \left.\times \int_{\mathrm{R}^{v} \times \mathrm{R}^{v}} d x_{4} S_{1}\left(t, x_{2}\right) \mathfrak{A}_{2}\left(t, x_{2}, x_{4}\right) S_{1}\left(t, x_{2}\right) F_{1}\left(t, x_{2}\right) S_{1}\left(t, x_{4}\right) F_{1}\left(t, x_{4}\right)\right] \\
& +\frac{1}{v^{2}} \frac{1}{2 !} \int_{\mathrm{R}^{2 v} \times \mathrm{R}^{2 v}} d x_{3} d x_{4} \mathfrak{A}_{4}\left(t, x_{1}, x_{2}, x_{3}, x_{4}\right) \prod_{i=1}^{4} S_{1}\left(t, x_{i}\right) F_{1}\left(t, x_{i}\right)+\cdots
\end{aligned}
$$

In terms of the operators $\hat{S}_{2}^{t}\left(x_{1}, x_{2}\right), \hat{\mathfrak{A}}_{\left|X_{i}\right|}\left(t, X_{i}\right)$ we obtain

$$
\begin{aligned}
& F_{2}\left(t, x_{1}, x_{2} \mid F_{1}(t)\right) \\
&= {\left[\hat{S}_{2}^{t}\left(x_{1}, x_{2}\right) \prod_{i=1}^{2} F_{1}\left(t, x_{i}\right)\right.} \\
&-\frac{1}{v}\left(\hat{S}_{2}^{t}\left(x_{1}, x_{2}\right) \int_{\mathrm{R}^{v} \times \mathrm{R}^{v}} d x_{3} \hat{\mathfrak{A}}_{2}\left(t, x_{1}, x_{3}\right) \prod_{i=1}^{3} F_{1}\left(t, x_{i}\right)\right. \\
&\left.+\hat{S}_{2}^{t}\left(x_{1}, x_{2}\right) \int_{\mathrm{R}^{v} \times \mathrm{R}^{v}} d x_{3} \hat{\mathfrak{A}}_{2}\left(t, x_{2}, x_{3}\right) \prod_{i=1}^{3} F_{1}\left(t, x_{i}\right)\right) \\
&+\frac{1}{v^{2}}\left(\hat { S } _ { 2 } ^ { t } ( x _ { 1 } , x _ { 2 } ) \int _ { \mathrm { R } ^ { 2 v } \times \mathrm { R } ^ { 2 v } } d x _ { 3 } d x _ { 4 } \left(\hat{\mathfrak{H}}_{2}\left(t, x_{2}, x_{3}\right) \hat{\mathfrak{U}}_{2}\left(t, x_{3}, x_{4}\right) \prod_{i=1}^{4} F_{1}\left(t, x_{i}\right)\right.\right. \\
&+\hat{\mathfrak{U}}_{2}\left(t, x_{2}, x_{3}\right) \hat{\mathfrak{U}}_{2}\left(t, x_{2}, x_{4}\right) \prod_{i=1}^{4} F_{1}\left(t, x_{i}\right)
\end{aligned}
$$




$$
\begin{aligned}
& \left.-\frac{1}{2 !} \hat{\mathfrak{A}}_{3}\left(t, x_{2}, x_{3}, x_{4}\right) \prod_{i=1}^{4} F_{1}\left(t, x_{i}\right)\right) \\
& +\hat{S}_{2}^{t}\left(x_{1}, x_{2}\right) \int_{\mathrm{R}^{v} \times \mathrm{R}^{v}} d x_{3} \hat{\mathfrak{A}}_{2}\left(t, x_{1}, x_{3}\right) \int_{\mathrm{R}^{v} \times \mathrm{R}^{v}} d x_{4} \hat{\mathfrak{A}}_{2}\left(t, x_{2}, x_{4}\right) \prod_{i=1}^{4} F_{1}\left(t, x_{i}\right) \\
& +\hat{S}_{2}^{t}\left(x_{1}, x_{2}\right) \int_{\mathrm{R}^{v} \times \mathrm{R}^{v}} d x_{3} \hat{\mathfrak{A}}_{2}\left(t, x_{2}, x_{3}\right) \int_{\mathrm{R}^{v} \times \mathrm{R}^{v}} d x_{4} \hat{\mathfrak{A}}_{2}\left(t, x_{1}, x_{4}\right) \prod_{i=1}^{4} F_{1}\left(t, x_{i}\right) \\
& +\hat{S}_{2}^{t}\left(x_{1}, x_{2}\right) \int_{\mathrm{R}^{2 v} \times \mathrm{R}^{2 v}} d x_{3} d x_{4}\left(\hat{\mathfrak{U}}_{2}\left(t, x_{1}, x_{3}\right) \hat{\mathfrak{A}}_{2}\left(t, x_{3}, x_{4}\right) \prod_{i=1}^{4} F_{1}\left(t, x_{i}\right)\right. \\
& +\hat{\mathfrak{A}}_{2}\left(t, x_{1}, x_{3}\right) \hat{\mathfrak{A}}_{2}\left(t, x_{1}, x_{4}\right) \prod_{i=1}^{4} F_{1}\left(t, x_{i}\right) \\
& \left.\left.\left.-\frac{1}{2 !} \hat{\mathfrak{A}}_{3}\left(t, x_{1}, x_{3}, x_{4}\right) \prod_{i=1}^{4} F_{1}\left(t, x_{i}\right)\right)\right)\right] \\
& +\left[\frac{1}{v} \int_{\mathrm{R}^{v} \times \mathrm{R}^{v}} d x_{3} \hat{\mathfrak{A}}_{3}\left(t, x_{1}, x_{2}, x_{3}\right) \prod_{i=1}^{3} F_{1}\left(t, x_{i}\right)\right. \\
& +\frac{1}{v^{2}}\left(\int_{\mathrm{R}^{v} \times \mathrm{R}^{v}} d x_{3} \hat{\mathfrak{A}}_{3}\left(t, x_{1}, x_{2}, x_{3}\right) \int_{\mathrm{R}^{v} \times \mathrm{R}^{v}} d x_{4} \hat{\mathfrak{H}}_{2}\left(t, x_{3}, x_{4}\right) \prod_{i=1}^{4} F_{1}\left(t, x_{i}\right)\right. \\
& +\int_{\mathrm{R}^{v} \times \mathrm{R}^{v}} d x_{3} \hat{\mathfrak{A}}_{3}\left(t, x_{1}, x_{2}, x_{3}\right) \int_{\mathrm{R}^{v} \times \mathrm{R}^{v}} d x_{4} \hat{\mathfrak{A}}_{2}\left(t, x_{1}, x_{4}\right) \prod_{i=1}^{4} F_{1}\left(t, x_{i}\right) \\
& \left.\left.+\int_{\mathrm{R}^{v} \times \mathrm{R}^{v}} d x_{3} \hat{\mathfrak{U}}_{3}\left(t, x_{1}, x_{2}, x_{3}\right) \int_{\mathrm{R}^{v} \times \mathrm{R}^{v}} d x_{4} \hat{\mathfrak{A}}_{2}\left(t, x_{2}, x_{4}\right) \prod_{i=1}^{4} F_{1}\left(t, x_{i}\right)\right)\right] \\
& +\frac{1}{v^{2}} \frac{1}{2 !} \int_{\mathrm{R}^{2 v} \times \mathrm{R}^{2 v}} d x_{3} d x_{4} \hat{\mathfrak{A}}_{4}\left(t, x_{1}, x_{2}, x_{3}, x_{4}\right) \prod_{i=1}^{4} F_{1}\left(t, x_{i}\right)+\cdots \\
& +
\end{aligned}
$$

In the representation of the functional $F_{2}\left(t, x_{1}, x_{2} \mid F_{1}(t)\right)$ we collect the terms of the same order in the density $\frac{1}{v}$. This yields the more compact form:

$$
\begin{aligned}
F_{2}( & \left.t, x_{1}, x_{2} \mid F_{1}(t)\right) \\
= & \hat{S}_{2}^{t}\left(x_{1}, x_{2}\right) \prod_{i=1}^{2} F_{1}\left(t, x_{i}\right) \\
& +\frac{1}{v} \int_{\mathrm{R}^{v} \times \mathrm{R}^{v}} d x_{3}\left[-\hat{S}_{2}^{t}\left(x_{1}, x_{2}\right)\left(\hat{\mathfrak{U}}_{2}\left(t, x_{1}, x_{3}\right)+\hat{\mathfrak{U}}_{2}\left(t, x_{2}, x_{3}\right)\right)\right.
\end{aligned}
$$




$$
\begin{aligned}
& \left.+\hat{\mathfrak{U}}_{3}\left(t, x_{1}, x_{2}, x_{3}\right)\right] \prod_{i=1}^{3} F_{1}\left(t, x_{i}\right) \\
& +\frac{1}{v^{2}} \int_{\mathrm{R}^{2 v} \times \mathrm{R}^{2 v}} d x_{3} d x_{4}\left[\left(\hat{\mathfrak{Q}}_{2}\left(t, x_{1}, x_{4}\right)+\hat{\mathfrak{U}}_{2}\left(t, x_{2}, x_{4}\right)+\hat{\mathfrak{A}}_{2}\left(t, x_{3}, x_{4}\right)\right)\right. \\
& \times \hat{S}_{2}^{t}\left(x_{1}, x_{2}\right)\left(\hat{\mathfrak{A}}_{2}\left(t, x_{1}, x_{3}\right)+\hat{\mathfrak{U}}_{2}\left(t, x_{2}, x_{3}\right)\right)-\hat{\mathfrak{A}}_{3}\left(t, x_{1}, x_{2}, x_{3}\right) \\
& -\frac{1}{2 !} \hat{S}_{2}^{t}\left(x_{1}, x_{2}\right)\left(\hat{\mathfrak{U}}_{3}\left(t, x_{1}, x_{3}, x_{4}\right)+\hat{\mathfrak{U}}_{3}\left(t, x_{2}, x_{3}, x_{4}\right)\right) \\
& \left.+\frac{1}{2 !} \hat{\mathfrak{U}}_{4}\left(t, x_{1}, x_{2}, x_{3}, x_{4}\right)\right] \prod_{i=1}^{4} F_{1}\left(t, x_{i}\right)+\cdots
\end{aligned}
$$

\section{Denote}

$$
\begin{aligned}
& \breve{\mathfrak{U}}^{(1)}(t)=-\hat{S}_{2}^{t}\left(x_{1}, x_{2}\right)\left(\hat{\mathfrak{U}}_{2}\left(t, x_{1}, x_{3}\right)+\hat{\mathfrak{A}}_{2}\left(t, x_{2}, x_{3}\right)\right)+\hat{\mathfrak{U}}_{3}\left(t, x_{1}, x_{2}, x_{3}\right), \\
& \breve{\mathfrak{U}}^{(2)}(t)=\left(\hat{\mathfrak{U}}_{2}\left(t, x_{1}, x_{4}\right)+\hat{\mathfrak{V}}_{2}\left(t, x_{2}, x_{4}\right)+\hat{\mathfrak{A}}_{2}\left(t, x_{3}, x_{4}\right)\right) \\
& \times \hat{S}_{2}^{t}\left(x_{1}, x_{2}\right)\left(\hat{\mathfrak{A}}_{2}\left(t, x_{1}, x_{3}\right)+\hat{\mathfrak{A}}_{2}\left(t, x_{2}, x_{3}\right)\right)-\hat{\mathfrak{U}}_{3}\left(t, x_{1}, x_{2}, x_{3}\right) \\
& -\frac{1}{2 !} \hat{S}_{2}^{t}\left(x_{1}, x_{2}\right)\left(\hat{\mathfrak{V}}_{3}\left(t, x_{1}, x_{3}, x_{4}\right)+\hat{\mathfrak{A}}_{3}\left(t, x_{2}, x_{3}, x_{4}\right)\right) \\
& +\frac{1}{2 !} \hat{\mathfrak{U}}_{4}\left(t, x_{1}, x_{2}, x_{3}, x_{4}\right)
\end{aligned}
$$

and so on. Then we obtain

(24)

$$
\begin{aligned}
& F_{2}\left(t, x_{1}, x_{2} \mid F_{1}(t)\right) \\
& =\hat{S}_{2}^{t}\left(x_{1}, x_{2}\right) \prod_{i=1}^{2} F_{1}\left(t, x_{i}\right) \\
& \quad+\frac{1}{v} \int_{\mathrm{R}^{v} \times \mathrm{R}^{v}} d x_{3} \breve{\mathfrak{H}^{(1)}}(t) \prod_{i=1}^{3} F_{1}\left(t, x_{i}\right) \\
& \quad+\frac{1}{v^{2}} \int_{\mathrm{R}^{2 v} \times \mathrm{R}^{2 v}} d x_{3} d x_{4} \breve{\mathfrak{H}^{(2)}}(t) \prod_{i=1}^{4} F_{1}\left(t, x_{i}\right)+\cdots \\
& =\hat{S}_{2}^{t}\left(x_{1}, x_{2}\right) \prod_{i=1}^{2} F_{1}\left(t, x_{i}\right) \\
& \quad+\sum_{n=1}^{\infty} \frac{1}{v^{n}} \int_{\mathrm{R}^{v n} \times \mathrm{R}^{v n}} d\left(X \backslash\left\{x_{1}, x_{2}\right\}\right) \breve{\mathfrak{H}^{(n)}}\left(t, X_{\left\{x_{1}, x_{2}\right\}}\right) \prod_{i=1}^{n+2} F_{1}\left(t, x_{i}\right) .
\end{aligned}
$$


We call equation (21) the generalized kinetic equation.

In view of estimate (5) and Theorem 4.1 the functional series in (17), and, more generally, the functional series for $F_{|Y|}\left(t, Y \mid F_{1}(t)\right)$ converges with respect to the norm of the space $L^{1}\left(\mathrm{R}^{v s} \times \mathrm{R}^{v s}\right)$, i.e., these functionals exist.

We omit, here, for the sake of conciseness, to write the explicit form of the $n$-th approximation $\breve{\mathscr{Y}}^{(\mathrm{n})}(t)$ in (17), since, to the aims of the present paper we only need the strong convergence of series (17).

\section{The existence theorem for the generalized kinetic equation}

The next theorem follows directly from Theorems 3.1 and 4.1.

THEOREM 6.1. Let $v, R$, and $r$ be strictly positive real numbers, and let $x$ and $z, 0<z<1$, be solutions to the equations

$$
\frac{e^{x}}{1-x}=\frac{2 R+r}{R+r}, \quad \text { and } \quad e^{z} \cdot \frac{1+z-z^{2}}{(1-z)^{2}}=2
$$

respectively. Suppose that $F_{1}(0) \in \mathrm{S}\left(F_{1}^{0}, R\right) \subset L_{0}^{1}\left(\mathrm{R}^{\nu} \times \mathrm{R}^{\nu}\right)$ and

$$
\frac{1}{v}<\frac{1}{R+r} \min \{x, z\} \text {. }
$$

Then there exist a unique strong, global in time, solution of the Cauchy problem for equation (21), which is given by strongly convergent series (15).

\section{Conclusion}

For low densities the Cauchy problem (1), (2) for the BBGKY hierarchy of equations with initial data satisfying the factorization property is reduced to the corresponding initial value problem for the generalized kinetic equation (21).

Thus, the generalized kinetic equation in explicit form is obtained for symmetric system of many particles interacting via a pair potential by using a solution of the Cauchy problem for the BBGKY hierarchy in the form of cumulant representation.

\section{REFERENCES}

1. Bogolyubov, N. N., Problems of a Dynamical Theory in Statistical Physics (Russian), Gosudarstv. Izdat. Tehn.-Teor. Lit., Moscow 1946.

2. Borgioli, G., Gerasimenko, V., Lauro, G., Derivation of a discrete Enskog equation from the dynamics of particles, Rend. Sem. Mat. Univ. Politec. Torino 56 (1998), 59-69.

3. Cercignani, C., Gerasimenko, V. I., Petrina, D. Ya., Many-Particle Dynamics and Kinetic Equations, Math. Appl. 420, Kluwer, Dordrecht 1997. 
4. Gerasimenko, V. I., Petrina, D. Ya., The generalized kinetic equation generated by the BBGKY hierarchy, Ukraïn. Fiz. Zh. 43 (1998), 697-702.

5. Gerasimenko, V. I., Ryabukha, T. V., Stashenko, M. O., On the structure of expansions for the BBGKY hierarchy solutions, J. Phys. A 37 (2004), 9861-9872.

6. Petrina, D. Ya., Gerasimenko, V. I., Mathematical description of the evolution of the state of infinite systems of classical statistical mechanics (Russian), Uspekhi Mat. Nauk. 38:5 (1983), 3-58.

7. Stashenko, M. A., Gubal', G. N., Existence theorems for the solution of an initial value problem for a chain of Bogolyubovequations in the space of sequences of bounded functions (Russian), Sib. Mat. Zh. 47 (2006), 188-205 (English transl.: Siberian Math. J. 47 (2006), 152-168).

LUTSK NATIONAL TECHNICAL UNIVERSITY

COMPUTER SCIENCE AND INFORMATION TECHNOLOGIES FACULTY

75 LVIVS'KA STR.

LUTSK 43018

UKRAINE

E-mail: gngu@mail.ru 